\title{
TLR Agonist CADI-05
}

National Cancer Institute

\section{Source}

National Cancer Institute. TLR Agonist CADI-05. NCI Thesaurus. Code C77884.

A poly-Toll-like receptor (TLR) agonist polyantigenic vaccine containing heat killed

Mycobacterium indicus pranii (Mycobacterium w or Mw) with potential

immunostimulating and antineoplastic activities. Upon administration, poly-TLR agonist polyantigenic vaccine activates a number of TLRs, which may result in macrophage and plasmacytoid dendritic cell (pDC) stimulation; secretion of interferon alpha; production of pro-inflammatory cytokines; upregulation of co-stimulatory molecules, enhanced T and B-cell stimulatory responses; T cell proliferation, and a Th1 immune response. TLRs are transmembrane receptors that recognize structurally conserved microbial molecules such as bacterial cell-surface lipopolysaccharides (LPS), lipoproteins, lipopeptides, lipoarabinomannan and flagellin, among others; immune responses stimulated by TLR activation may result in antineoplastic effects. 